\title{
Neuroendocrine tumors: a focus on liver metastatic lesions
}

\section{Georgios S. Limouris*}

Athens University Medical Faculty, Nuclear Medicine Division, Radiology Department, Aretaieion University Hospital, Athens, Greece

\section{Edited by:}

Karel Pacak, National Institutes of Health, USA

\section{Reviewed by:}

Karel Pacak, National Institutes of Health, USA

Henri Timmers, Radboud University Nijmegen Medical Centre,

Netherlands

*Correspondence:

Georgios S. Limouris, Athens University Medical Faculty, Nuclear Medicine Division, Radiology Department, Aretaieion University Hospital, Vas Sophias Avenue 76, Athens 11528, Greece.

e-mail: nucleard@aretaieio.uoa.gr
Transhepatic radionuclide infusion has been introduced as a new treatment approach for unresectable liver neuroendocrine metastatic lesions with the prerequisite of a positive In-111 Pentetreotide (Octreoscan). Patients with multiple liver neuroendocrine metastases can be locally treated after selective hepatic artery catheterization and infusion of radiolabeled somatostatin analogs, and in case of extra-hepatic secondary spread, after simple i.v. application. According to the world wide references, the average dose per session to each patient is $6.3 \pm 0.3 \mathrm{GBq}(\sim 160-180 \mathrm{mCi})$ of In-111-DTPA-Phe1-Pentetreotide, 10- to 12-fold in total, administered monthly or of $4.1 \pm 0.2 \mathrm{GBq}(\sim 105-116 \mathrm{mCi})$ of Y-90 DOTA TOC, threefold in total, or of $7.0 \pm 0.4 \mathrm{GBq}(\sim 178-200 \mathrm{mCi})$ of Lu-177 DOTA TATE, fourfold to sixfold in total (the choice of which being based on the tumor size, assessed by CT or MRI). Follow-up at monthly intervals has to be performed by means of ultrasonography (US). Treatment response has to be assessed according to the WHO criteria (RECIST or SWOG).

Keywords: radiopeptide therapy, neuroendocrine tumors, Y-90 DOTA TOC Lu-177 DOTA TATE, In-111-DTPA-Phe1pentetreotide

\section{INTRODUCTION}

On the course of the development from a normal cell to a cancer one the overexpression of surface receptors consists a very important characteristic among a plethora of morpho-functional changes of these cells. This receptor overexpression provides the possibility to be used as a target site of corresponding peptides which, if appropriately radiolabeled, can be used for imaging and further shifting to treating purposes.

The therapeutic implementation of the radiolabeled peptides is based on: (i) their proven internalization into the cytoplasma and consequently (ii) their tight approach to the nucleus, in striking distance from the chromosomes, with DNA being the fatal target within the radiomolecular emission range (a necessary and obligatory assumption for the certain chromosomal destroy; Anderson et al., 1996; Tiensuu Janson et al., 2000; Buchegger et al., 2006).

\section{THE CONCEPT}

Since the discovery in the late 1970s by Guillemin of native tetradeca-peptide somatostatin (Guillemin and Gerich, 1976), five specific receptor subtypes have been discovered and cloned (Hoyer et al., 1995). However, their extremely short plasma halflife ( $<3 \mathrm{~min}$ ) inspired the synthesis of analogs with more favorable characteristics, that means a longer half-life and a marked smaller molecule. First synthetic analog of tetradeca-peptide somatostatin used to be octreotide, containing the bioactive core of the native molecule, which consists of eight amino-acids. Octreotide binds with relative high affinity $(22 \pm 3.6 \mathrm{nM})$ only to subtype II somatostatin receptors whereas with a lower one to subtypes III $(182 \pm 13 \mathrm{nM})$ and V $(237 \pm 52 \mathrm{nM}$; Reubi et al., 2000; Schmitt et al., 2001). Labeled with indium-111 (Octreoscan, Mallinckrodt, Petten, The Netherlands) it has been established as the radiopharmaceutical of choice for selective imaging of tissues and lesions positive mainly for somatostatin subtype II receptors. After i.v. application, Octreoscan is internalized into the tumor-cell by fluid-phase endocytosis, and degraded in two insoluble metabolites In-111 DTPA-D-Phe-Cys-OH and In-111 DTPA-D-Phe-OH (Anderson et al., 1996; De Jong et al., 1997; Bass et al., 1998) inside the lysosomes, close to the nucleus, where they are retained (De Jong et al., 1998; Janson et al., 2000). Thereafter, the empty receptor drives again to the cell membrane surface. Subsequently, the use of Octreoscan was extended with success for therapeutic purposes too, in particular for the confrontation of unresectable neuroendocrine tumors (i) due to the high efflux of indium-111 Auger and Internal Conversion electrons and (ii) the extremely high radioactive ratio of the tumor vs. the healthy peri-tumoral tissue (approximately 30:1).

\section{THE TUMOR CONFRONTATION}

First, in 1993, high doses of In-111-DTPA-Phe1-octreotide were used for the treatment of these type of tumors (Krenning et al., 1993, 1994) via antecubital intravenous infusions, exploiting the Auger (0.02-10 $\mu \mathrm{m}$ particle range), and Internal Conversion electron emission (200-550 $\mu \mathrm{m}$ particle range) of indium-111 (Adelstein, 1993; Krenning et al., 1993, 1994). However, because of the too small particle range and therefore the lower tissue penetration, ${ }^{111}$ In-labeled peptides could not be the considered as the ideal radiopharmaceutical for peptide receptor radionuclide therapy (PRRT) and their use were monitored for liver metastasized tumors of smaller than $20 \mathrm{~mm}$ diameter, and strictly intraarterially, after catheterization of the hepatic artery (Limouris et al., 2008). This application way is directly related to the rheology of the administration route, as the distance between the point of the i.a. infusion (the femoral artery) up to the hepatic artery/feeding tumor arterial net (first pass extraction) is short, and promptly trapped by the tumor somatostatin receptors, overpassing the renal arteries. This is in contrast with the long distance 
venous way (antecubital up to the pulmonary vein net) in case of i.v. implementation. The aforementioned result in a maximization of the absorbed activity rate onto the metastatic liver lesions compared to that calculated after an i.v. infusion, achieving a larger tumor burden with the lowest possible delivered dose to the kidneys (Limouris et al., 1999, 2008).

In the late 1990s, intensive work and investigations in radiochemistry considered yttrium-90 to be more suitable and effective for therapeutic use compared to indium-111 because of its energetic $\beta$-particles $\left(E_{\max }=2.27 \mathrm{MeV}\right)$ and long range $\left[R_{95}=5.94 \mathrm{~mm}\left(R_{95}\right.\right.$ being the distance within which the $\beta$ particle transfers $95 \%$ of its energy to the target tissue)] which allows "cross-fire" irradiation (Paganelli et al., 2001). Coupled with DOTA chelator, binds with an affinity of $11 \pm 1.7,389 \pm 135$, and $114 \pm 29 \mathrm{nM}$ to subtype II, III, and V somatostatin receptors respectively (Reubi et al., 2000; Schmitt et al., 2001). Radiotherapeutic use of Y-90DOTATOC leads to a higher and more evenly distributed radiation dose to the tumor because of its larger particle range $\left(R_{\max }: 11 \mathrm{~mm}\right)$ and tissue penetration. Tumors with an in homogeneous distribution of receptors seem to respond favorably to PRRT treatments with Y-90 DOTATOC (De Jong et al., 1997; Paganelli et al., 2001; Bodei et al., 2004).

However, the use of yttrium-90 did not fully satisfy the therapists involved in the treatments of neuroendocrine malignancies due to the lack of $\gamma$ emission that highly facilitates the dosimetric work. The scientific efforts and pursuances lead to the development of a third generation radiopeptides, labeled with a longer half-life and a $\gamma$ emission nuclide as is the case with Lu-177 DOTA TATE. With a $\mathrm{E} \beta$ max of $0.497 \mathrm{MeV}$, a $\mathrm{E} \gamma$ of $113 \mathrm{KeV}(6.4 \%)$, and $208 \mathrm{KeV}(11.0 \%)$ a penetration range of $2 \mathrm{~mm}$ and a 6.7 -days half-life Lu-177 DOTA TATE binds with an affinity of $1.5 \pm 0.4$, $453 \pm 176$, and $547 \pm 160 \mathrm{nM}$ to subtype II, III, and V somatostatin receptors respectively (Reubi et al., 2000; Kwekkeboom et al., 2001, 2003, 2005; Schmitt et al., 2001; Seregni et al., 2010) (Figure 1).

The treatment of liver neuroendocrine metastases is still a major doctor's dilemma because the majority of patients present with extensive unresectable disease. Apart from surgical resection which is the treatment of choice, systemic therapy, hepatic artery (chemo-) embo-lization, and radiofrequency ablation claim proved clinical benefit. Patients, objective to surgery with curative intention are few, and a complete resection still show a low (30\%) overall survival rate. In systemic therapy the use of intravenous chemotherapy is not leading to the best expected results due to the diverse variables, including type of chemotherapy, stage of disease, progression, and toxicity. In our Institution the concept of isolated liver radionuclide perfusion was firstly developed in an hepatoma case, were In-111 labeled somatostatin, after selective catheterization of the hepatic artery, was injected (Limouris et al., 1998, 1999). Thus, by this way, a markedly higher radiation amount within the liver nodule can be deposited and in

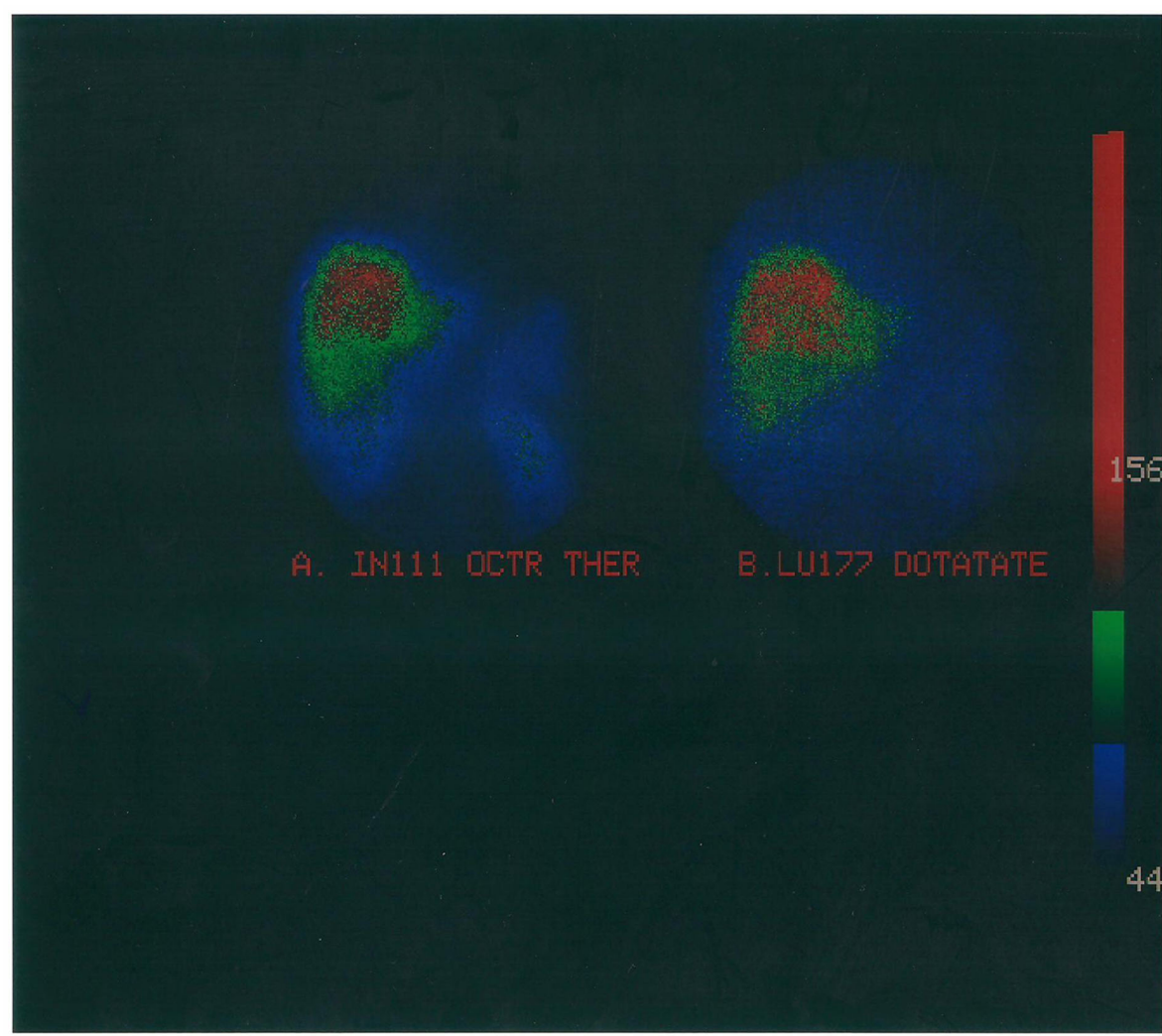

FIGURE 1 | Radiomolecular combined therapy of multiple liver metastases originated from a resected pancreatic neuroendocrine tumor treated with high doses of (A). In-111 Pentetreotide and (B) Lu-177 DOTATATE. 
parallel with the lowest possible, extra-tumoral radiobiological burden mainly, in regard to the kidneys (critical organ). Purpose of this aforementioned administration procedure was to introduce transhepatic radionuclide infusion (THRI) as a treatment option modality for unresectable, multiple, small, or moderate in size, liver metastases, routinely, along with a nephroprotective scheme comprising amino-acid solution $(1000 \mathrm{~mL})$, fallowed by $50 \mathrm{mg}$ DTPA in $250 \mathrm{~mL}$ normal saline, in drip infusion ("The Aretaieion Protocol").

\section{NON-RADIOMOLECULAR TREATMENT OPTIONS}

From the pharetra of loco-regional ablation techniques, cryosurgery is an open abdominal surgery procedure with risks and complications, needing special equipment with cryoprobes working by means of supercooled liquid nitrogen and argon gas. (Chemo-) embo-lization and percutaneous radiofrequency ablation are two minimally invasive procedures which compared to cryotherapy, are usually done in a conventional radiology unit. For the latter two, the number of liver lesions under treatment should be less than 3 , a prerequisite not to be ignored, since the majority of the neuroendocrine liver metastases are multiple.

\section{PERSPECTIVES}

Transhepatic radionuclide infusion presents a new viewpoint on an important area of research, aiming to consist the treating modality of preference for the confrontation of unresectable neuroendocrine malignancies. Comparing and evaluating the worldwide experience of the rather few international centers of excellence, routinely dealing with radiopeptide therapy, the following perspectives might optimize their application.

\section{IMPLEMENTATION IN COCKTAILS}

Applied in combination (i.e., indium-111/lutetium-177 or yttrium-90/lutetium-177) and exploiting the different radionuclide emission ranges, irradiating the tumor-cell in different loci from the point of their uptake (receptors), they increase their destruction capability (Limouris et al., 2008; Seregni et al., 2010).

\section{INTRA-ARTERIAL VS. INTRAVENOUS APPLICATION}

Where the i.a. way is possible (liver or brain lesions) it has to be preferred because the amount of the tumor absorbed dose is

\section{REFERENCES}

Adelstein, S. J. (1993). The Auger process: a therapeutic promise? AJR Am. J. Roentgenol. 60, 707-713.

Anderson, P., Forssel-Aronsson, E., Johanson, V., Wängberg, B., Nilsson, O., Fjälling, M., and Ahlman, $\mathrm{H}$. (1996). Internalization of In-111 in to human neuroendocrine tumor cells after incubation with indium-111-DTPA-D-Phe1 octreotide. J. Nucl. Med. 37, 2002-2006.

Bass, L. A., Lanahan, M. V., Duncan, J. R., Erion, J. L., Srinivasan, A., Schmidt, M. A., and Anderson, C. J. (1998). Identification of the soluble in vivo metabolites of indium-111-

higher. This happens because the radiopharmaceutical covers a much longer distance to reach the target-organ (tumor DNA), initiating the radiopeptide infusion after venous catheterization, since an important part of it, is taken up from the traversing tissues. Such tissues are the kidneys which for the THRI consist the critical organ. It is proved (Kontogeorgakos et al., 2006; Limouris et al., 2008) that the kidney biological burden is in a more than $40 \%$ decreased compared to that calculated in i.v. infusions.

\section{THRI BEFORE SURGICAL EXERESIS}

Large volume neuroendocrine tumors over $6 \mathrm{~cm}$ in diameter (about more than $60 \mathrm{~g}$ ) is extremely difficult to be managed. Their treatment initialization with surgical excision lurks a potential dissemination tumor-cell-spread. On the contrary, THRI alone, cannot provide the necessary absorbed dose to them in order to obtain a satisfactory response (partial or complete) due to (i) the receptor heterogeneity of the large lesions, not achieving the expected high absorbed doses and (ii) the kidney radiobiological overburden, rendering in a mass, as forbidden, for any further treatment continuation. Conventional intravenous therapy regarding large volume tumors is doubtless insufficient. Therefore, initializing the treatment course by means of 3-4 cycles of THRI, prior to the surgical excision, the dissemination danger can be potently minimized and the forthcoming surgical intervention more safely implemented.

Finally, it would be an omission to not report on data regarding the time interval between the initialization of a given treatment up to any regression time observed to the treated tumors, i.e., the progression-free survival (PFS), considering the neuroendocrine disease as a chronic illness. In studies by Kwekkeboom et al. (2008) with Lu-177 DOTA TATE, the median PFS was 40 months. In studies by Cwikla with Y-90-DOTA TATE, the median PFS was 17 months, whereas in studies by Valkema with Y-90 DOTATOC the median PFS was reported to be 30 months (Valkema et al., 2003; Cwikla et al., 2010).

Newer strategies to augment the therapeutic potential of PRRT in combination with radiosensitizing chemotherapy or biomolecular targeted agents, are so far under investigation.

Guttmejer, A., Jeziorski, K. G., Mikolajczak, R., Pawlak, D., Stepien, K., and Walecki, J. (2010). Efficacy of radionuclide treatment DOTATATE Y-90 in patients with progressive metastatic gastroenteropancreatic neuroendocrine carcinomas (GEP-NETs): a phase II study. Ann. Oncol. 21, 787-794.

De Jong, M., Bakker, W. H., Krenning, E. P., Breeman, W. A. P., Van der Pluijm, M. E., Bernard, B. F., Visser, T. J., Jermann, E., Béhé, M., Powell, P., Mäcke, H. R., and Mäcke, H. R. (1997). Yttrium-90 and indium-111 labelling, receptor binding and biodistribution of [DOTA0,D-Phe1,Tyr3] octreotide,

a promising somato-statin for analogue radionuclide therapy. Eur. J. Nucl. Med. 24, 368-371.

De Jong, M., Bernard, B. F., De Bruin, E., Van Gameren, A., Bakker, WH., Visser, T. J., Mäcke, H. R., and Krenning, E. P. (1998). Internalization of radiolabelled $\left[\mathrm{DTPA}^{\circ}\right]$ octreotide and [DOTA $^{\circ}$, Tyr3] octreotide: peptides for somatostatin receptortargeted scintigraphy and radionuclide therapy. Nucl. Med. Commun. 19, 283-288.

Guillemin, R., and Gerich, J. E. (1976). Somatostatin: physiological and clinical significance. Annu. Rev. Med. 27, 379-388.

Hoyer, D., Bell, G. I., Berelowitz, M., Epelbaum, J., Feniuk, W., Humphrey, P. P. A., O’Carroll, A.-M., Patel, Y. C., 
Schonbrunn, A. J., Taylor, E., and Reisine, T. (1995). Classification and nomenclature of somatostatin receptors. Trends Pharmacol. Sci. 16, 86-88.

Janson, E. T., Westlin, J.-E., Öhrvall, U., Öberg, K., and Lukinius, A. (2000). Nuclear loca-lization of 111 In after intravenous injections of [111In-DTPA-DPhe1]-octreotide in patients with neuroendocrine tumours. J. Nucl. Med. 41, 1514-1518.

Kontogeorgakos, D., Dimitriou, P., Limouris, G. S., and Vlachos, L. J. (2006). Patient specific dosimetry calculations during therapy with In-111-DTPA-D-Phe1-octreotide infusions after catheterization of the hepatic artery using mathematical models of different anatomical sizes. J. Nucl. Med. 47, 1476-82.

Krenning, E. P., Kooij, P. P. M., Bakker, W. H., Breeman, W. A. P., Postema, P. T. E., Kwekkeboom, D. J., Oei, H. Y., De Jong, M., Visser, T. J., Reijs, A. M., and Lamberts, S. W. J. (1994). Radiotherapy with a radiolabeled somatostatin analogue, [111In-DTPA-D Phe1]-octreotide; a case history. Ann. N. Y. Acad. Sci. 733, 496-506.

Krenning, E. P., Kwekkeboom, D. J., Bakker, W. H., Breeman, W. A., Kooij, P. P., Oei, H. Y., Van Hagen, M., Postema, P. T. E., Reubi, J. C., Visser, T. J., Reijs, A. M., Hofland, L. J., Koper, J. W., and Lamberts, S. W. J. (1993). Somatostatin receptor scintigraphy with [111In- DTPA-DPhel]- and [123I-Tyr3]-octreotide: the Rotterdam experience with more than 1000 patients. Eur. J. Nucl. Med. 20, 716-731.

Kwekkeboom, D. J., Bakker, W. H., Kam, B. L., Teunissen, J. J. M., Kooij, P. P. M., De Herder, W. W., Feelders, R. A., van Eijck, C. H., de Jong, M., Srinivasan, A.,
Erion, J. L., and Krenning, E. P. (2003). Treatment of patients with gastroentero-pancreatic (GEP) tumours with the novel radiolabelled somatostatin analogue [177Lu-DOTA ${ }^{\circ}$, Tyr3] octreotate. Eur. J. Nucl. Med. 30, 417-422.

Kwekkeboom, D. J., Bakker, W. H., Kooij, P. P. M., Konijnenberg, M. W., Srinivasan, A., Erion, J. L., Schmidt, M. A., Bugaj, J. L., de Jong, M., and Krenning, E. P. (2001). Lu177-DOTA Tyr octreotate: comparison with In-111-DTPA-octreotide in patients. Eur. J. Nucl. Med. 28, 1319-1325.

Kwekkeboom, D. J., de Herder, W. W., Kam, B. L., van Eijck, C. H., van Essen, M., Kooij, P. P., Feelders, R. A., van Aken, M. O., and Krenning, E. P. (2008). Treatment with the radiolabeled somatostatin analog [177 Lu-DOTA 0,Tyr3] octreotate: toxicity, efficacy, and survival. J. Clin. Oncol. 26, 2124-2130.

Kwekkeboom, D. J., Mueller-Brand, J., Paganelli, G., Anthony, L. B., Pauwels, S., Kvols, L. K., O'dorisio, T. M., Valkema, R., Bodei, L., Chinol, M., Maecke, H. R., and Krenning, E. P. (2005). Overview of results of peptide receptor radionuclide therapy with 3 radiolabeled somatostatin analogs. J. Nucl. Med. 46(Suppl. 1), 62-66.

Limouris, G. S., Chatziioannou, A., Kontogeorgakos, D., Mourikis, D., Lyra, M., Dimitriou, P., Stavraka, A., Gouliamos, A., and Vlahos, L. (2008). Selective hepatic arterial infusion of In111-DTPA-Phe1-octreotide in neuroendocrine liver metastases. Eur. J. Nucl. Med. Mol. Imaging 35, 1827-1837.

Limouris, G. S., Lyra, M., Skarlos, D., Chatziioannou, A., Gouliamos, A., Moulopoulou, A., and Vlahos, L. (1999). "Auger and conversion electron therapy with In-111 pentetreotide in hepatocellular carcinoma," in Radioactive Isotopes in Clinical Medicine and Research, eds H. Bergmann, H. Koehn, and H. Sinzinger (Boston: Birkhäuser), 551-554.

Limouris, G. S., Lyra, M., Voliotopoulos, V., Frantzis, A. P., Gouliamos, A., Chatziioannou, A., Vlahos, L., and Stavrala, A. (1998). "On therapeutic application of [In-111-DTPAD-Phe] octreotide in hepatocellular carcinoma," in Radionuclides for Oncology; Current Status and Future Aspects, eds G. S. Limouris, S. K. Shukla, and H. F. Bender, H.-J. Biersack (Athens: Mediterra Pbls), 53-55.

Paganelli, G., Zoboli, S., Cremonesi, M. Bodei, L., Ferrari, M., Grana, C., Bartolomei, M., Orsi, F., De Cicco, C., Mäcke, H. R., Chinol, M., and de Braud, F. (2001). Receptor-mediated radiotherapy with 90Y-DOTA-DPhe1-Tyr3-octreotide. Eur. J. Nucl. Med. 28, 426-434.

Reubi, J.-C., Schär, J.-C., Waser, B., Wenger, S., Heppeler, A., Schmitt, J. S., and Mäcke, H. R. (2000). Affinity profiles for human somatostatin receptor subtypes SST1-SST5 of somatostatin radiotracers selected for scintigraphic and radiotherapeutic use. Eur. J. Nucl. Med. 27, 273-282.

Schmitt, J. S., Wild, D., Ginj, M., Reubi, J. C., Waser, B., De Jong, M., Bernard, H. F., Krenning, E. P., and Maecke, H. R. (2001). DOTA-NOC, a high affinity ligand of the somatostatin receptor subtypes 2,3 and 5 for radiotherapy. J. Labelled Comp. Radiopharm. 44, s697-s699.

Seregni, E., Maccauro, M., Coliva, A., Castellani, M. R., Bajetta, E., Aliberti, G., Vellani, C., Chiesa, C., Martinetti, A., Bogni, A., and Bombardieri, E. (2010). Treatment with tandem [(90)Y]DOTA-TATE and [(177)Lu] DOTA-TATE of neuroendocrine tumors refractory to conventional therapy: preliminary results. Q. J. Nucl. Med. Mol. Imaging. 54, 84-91.

Tiensuu Janson, E., Westlin, J.-E., Öhrvall, U., Öberg, K., and Lukinius, A. (2000). Nuclear localization of In-111 after intravenous injection of In-111 DTPA-D-Phel octreotide in patients with neuroendocrine tumors. J. Nucl. Med. 41, 1514-1518.

Valkema, R., Pauwels, S., Kvols, L., Jamar, F., Barone, R., Bakker, W. H. Lasher, J., and Krenning, E. P. (2003). Long-term follow-up of a phase 1 study of peptide receptor radionuclide therapy (PRRT) with [90YDOTA0,Tyr3] octreotide in patients with somatostatin receptor positive tumours. Eur. J. Nucl. Med. Mol. Imag. 30 (Suppl. 2), S232 (abstract).

Conflict of Interest Statement: The author declares that the research was conducted in the absence of any commercial or financial relationships that could be construed as a potential conflict of interest.

Received: 04 August 2011; paper pending published: 28 August 2011; accepted: 14 February 2012; published online: 28 February 2012.

Citation: Limouris GS (2012) Neuroendocrine tumors: a focus on liver metastatic lesions. Front. Oncol. 2:20. doi: 10.3389/fonc.2012.00020

This article was submitted to Frontiers in Cancer Imaging and Diagnosis, a specialty of Frontiers in Oncology.

Copyright () 2012 Limouris. This is an open-access article distributed under the terms of the Creative Commons Attribution Non Commercial License, which permits non-commercial use, distribution, and reproduction in other forums, provided the original authors and source are credited. 